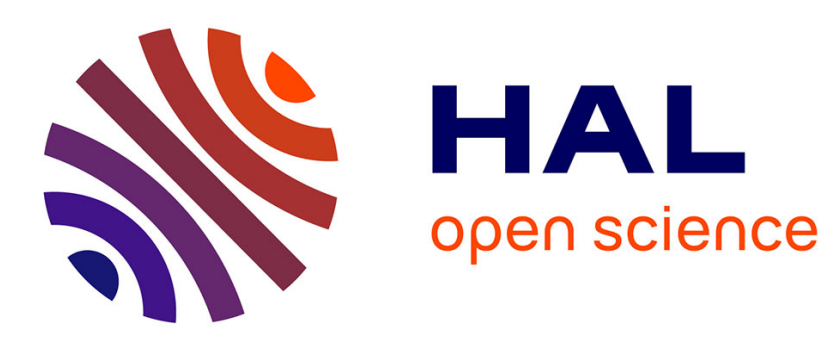

\title{
Conseils de Prud'hommes, Syndicats et resolution des litiges : une analyse economique
}

\author{
Claudine Desrieux, Romain Espinosa
}

\section{To cite this version:}

Claudine Desrieux, Romain Espinosa. Conseils de Prud'hommes, Syndicats et resolution des litiges: une analyse economique. Les Cahiers de la justice, 2015, 2015 (4), pp.533-545. halshs-01793459

\section{HAL Id: halshs-01793459 \\ https://shs.hal.science/halshs-01793459}

Submitted on 16 May 2018

HAL is a multi-disciplinary open access archive for the deposit and dissemination of scientific research documents, whether they are published or not. The documents may come from teaching and research institutions in France or abroad, or from public or private research centers.
L'archive ouverte pluridisciplinaire HAL, est destinée au dépôt et à la diffusion de documents scientifiques de niveau recherche, publiés ou non, émanant des établissements d'enseignement et de recherche français ou étrangers, des laboratoires publics ou privés. 


\title{
Conseils de Prud'hommes, Syndicats et résolution des litiges : une analyse économique
}

\author{
par Claudine Desrieux ${ }^{1}$ et Romain Espinosa ${ }^{2}$
}

\begin{abstract}
Singuliers dans le paysage juridictionnel français, les conseils de prud'hommes règlent les différends liés aux contrats de travail entre employeurs et salariés. La justice est exercée par des conseillers prudhommaux, proposés par des organisations syndicales, et élus au niveau de chaque juridiction par les salariés et les employeurs suivant un principe de paritarisme. La composition syndicale de chaque conseil varie donc d'une juridiction à l'autre. L'article suivant s'intéresse à l'influence de la composition syndicale des conseils sur les stratégies des parties et l'issue des litiges. Les résultats sont commentés par Florence Audier, économiste.
\end{abstract}

French labor courts deal with conflicts between employers and employees in the private sector and are specialized courts. Judges are elected on a parity basis every five years within lists proposed by both workers' unions and employers' federations. Elections are held at the local level so that each court has a different composition regarding the scores obtained during the election. The following article wonders whether this composition influences the strategies of the parties at court and the outcomes of the claims. Results are commented by Florence Audier, an economist.

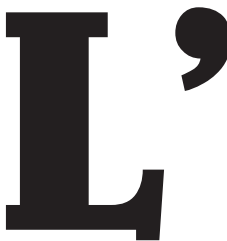

analyse économique du droit s'est progressivement constituée en champ disciplinaire distinct depuis les années 1950. D'abord impulsé par des chercheurs d'universités amé-

1. Claudine Desrieux, Maître de conférences en sciences économiques, CRED (TEPP) Université Paris II Panthéon-Assas, 12 Place du Panthéon, 75005 PARIS, claudine.desrieux@u-paris2.fr

2. Romain Espinosa, Doctorant contractuel en sciences économiques, CRED (TEPP) Université Paris II Panthéon-Assas, 12 Place du Panthéon, 75005 PARIS, romain.espinosa@u-paris2.fr.

Les auteurs adressent leurs plus vifs remerciements au Ministère de la Justice, et en particulier à M. Camus et à Mme Lixi, pour ricaines autour de R. Coase, G. Calabresi, et G. Becker, ce courant d'analyse s'est ensuite répandu dans de nombreuses autres universités. L'objectif de l'analyse économique du droit est d'étudier le comportement des agents économiques, en considérant que ce

leur avoir permis d'accéder aux données nécessaires à leur travail. Les auteurs expriment également leur profonde gratitude au personnel du service statistique du Ministère ainsi que celui du CASD (Centre d'accès sécurisé aux donnée) pour avoir permis l'usage de ces données dans un environnement sécurisé. Le Ministère du travail, de l'emploi et du dialogue social est également remercié pour les données concernant les résultats des élections prud'homales. 
Dossier Conseils de Prud'hommes, Syndicats et résolution des litiges : une analyse économique

comportement est en partie influencé par les règles de droit, et qu'en retour, les agents peuvent également contribuer à façonner ces règles. Les domaines d'application de l'analyse économique du droit sont donc très nombreux et divers : l'étude des droits de propriété, des règles de responsabilité, des politiques de prévention des activités illégales, ou encore les règles régissant les activités économiques sont autant d'objets de recherches. ${ }^{3}$ L'intérêt porte à la fois sur la nature des règles, mais aussi sur les conditions de leurs applications. Face à ces thèmes «juridiques », l'apport des économistes est essentiellement méthodologique: il s'agit de proposer une grille de lecture différente, et de contribuer ainsi au débat pluridisciplinaire. Le raisonnement économique se bâtit en effet sur une série d'hypothèses permettant d'établir des propositions théoriques. Ces dernières sont ensuite soumises à des «tests empiriques", c'est-à-dire confrontées aux données statistiques, pour voir dans quelle mesure elles sont corroborées (ou non) par les faits. Il s'agit donc d'utiliser les « outils» développés par les économistes pour les appliquer à des problématiques juridiques, et de contribuer ainsi au dialogue interdisciplinaire. En effet, les propositions ainsi avancées par les économistes appellent à être discutées et confrontées à l'expérience

3. Sur le pluralisme des approches en analyse économique du droit, voir l'article: Deffains B., Ferey S. (2007), «Théorie du droit et analyse économique », Droits, juillet, pp. 223-254.

4. Ce travail est disponible sur demande aux auteurs. II est coécrit par Claudine Desrieux et Romain Espinosa, rédigé en anglais et a pour titre "Litigants' strategies in elected courts: Evidence from french labor courts » (2015).

5. Nous nous cantonnerons ici à un bref rappel de contributions antérieures proches de notre travail. Pour plus d'informations des chercheurs d'autres disciplines et à celle des praticiens pour pouvoir s'enrichir. Cette démarche a animé un récent travail que nous avons mené sur la justice prud'homale en France. ${ }^{4}$ Nous proposons dans cet article d'en présenter et discuter les principaux résultats. À travers cette étude, nous nous sommes inscrits dans une littérature économique récente, s'intéressant aux déterminants des décisions de justice. Cette littérature a d'abord été impulsée par des travaux sur le fonctionnement de la cour suprême aux États-Unis. ${ }^{5}$ Ils se sont par la suite étendus au fonctionnement de nombreuses autres juridictions, notamment européennes. Ainsi, une première étude ${ }^{6}$ a été menée par A. Ichino, M. Polo et E. Rettore en 2003 sur des données issues d'une banque italienne ayant des succursales sur tout le territoire italien. Leur étude a mis en avant le rôle de l'environnement macroéconomique sur les décisions de justice relatives au droit du travail. Sur la période 1979-1995, ces auteurs ont examiné 1862 cas de licenciements pour faute de salariés masculins de cette banque. Leur principal résultat est de montrer que dans un contexte de chômage élevé, on constate des motifs de fautes plus sévères et pourtant un taux d'acceptation de la demande des salariés plus élevé. Ces résultats suggèrent que les juges intègrent l'envi-

sur les travaux portant sur la Cour Suprême, voir Espinosa (2015) dans ce numéro, et pour d'autres travaux sur les prises de décision en justice, voir Philippe (2015), également dans ce numéro.

6. Ichino, A., Polo, M., and Rettore, E. (2003). Are Judges Biased by Labor Market Conditions? European Economic Review, 47 (5):913-944. 
ronnement économique dans les décisions rendues: dans un contexte de difficultés économiques, ils auraient tendance à plus accepter les demandes des salariés contestant leur licenciement que lorsque le chômage est faible. Dans la lignée de ce travail, I. Marinescu a proposé une étude ${ }^{7}$ en 2011 sur 1311 cas de contestations de licenciements au Royaume-Uni, entre janvier 1990 et octobre 1991. Pour ces cas, Marinescu dispose des caractéristiques des salariés et des employeurs, des motifs de la demande des salariés, des montants de compensation accordée, et de données macroéconomiques (taux de chômage et taux de faillite des entreprises). Son étude empirique montre que les salariés (demandeurs) ayant retrouvé un emploi au moment de l'audience ont moins de chance de voir leur demande acceptée lorsque le taux de chômage et le taux de faillite des entreprises sont élevés. L'interprétation de ce résultat semble donc aller en sens opposé à celui d'Ichino et ses coauteurs, tout en montrant que l'environnement économique n'est pas neutre sur les décisions prononcées. La raison de cette divergence peut être due au fait que les données d'Ichino n'étaient relatives qu'à une seule entreprise, et que les deux études portent sur un cadre institutionnel issu de deux pays différents. Une autre contribution est celle de H. Berger et M. Neugart (2011). ${ }^{8}$ La perspective de ces auteurs est légèrement différente: leur intérêt ne porte pas sur l'en-

7. Marinescu, I. (2011). Are Judges Sensitive to Economic Conditions? Evidence from UK Employment Tribunals, Industrial and Labor Relations Review, 64(4):673-698. vironnement économique, mais sur les biais de nomination des juges exerçant le droit du travail en Allemagne. En effet, les cours d'appel ont des juges nommés par des représentants politiques. Ces auteurs montrent que lorsque la proportion de juges nommés par des gouvernements conservateurs augmente, on observe moins de litiges dès la première instance, plus de négociation, et plus d'appels.

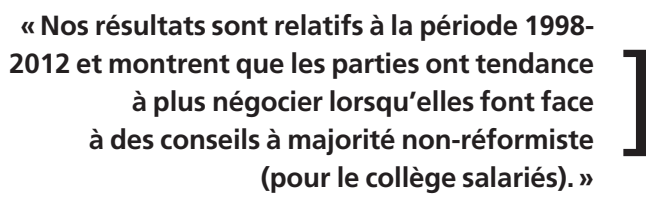

Notre étude s'inscrit dans cette série de travaux. Appliquée au cadre français, elle a pour objectif de déterminer si la composition syndicale des conseils de prud'hommes influence le comportement des parties. Nos résultats sont relatifs à la période 1998-2012 et montrent que les parties ont tendance à plus négocier lorsqu'elles font face à des conseils à majorité non-réformiste (pour le collège salariés). Dans ces conseils, on constate également un plus fort taux de recours au départage. Cependant, nous ne trouvons pas que le taux d'acceptation des litiges (à l'étape du bureau des jugements ou bien au départage) varie de manière significative entre les conseils à majorité non-réformiste et les autres. De même, le volume des litiges (i.e. le nombre de litiges portés devant

8. Berger, H. and Neugart, M. (2011). Labor Courts, Nomination Bias, and Unemployment in Germany. European Journal of Political Economy, 27. 
chaque conseil) ne semble pas être influencé par la composition syndicale des conseils.

Nous nous proposons ci-dessous de détailler ces résultats. Dans un premier temps, un bref rappel du fonctionnement de la juridiction prud'homale est réalisé. Ensuite, nous détaillons le raisonnement économique fondant notre intuition. Dans un troisième temps, nous présentons les résultats empiriques de notre étude, pour terminer sur une discussion de nos résultats.

\section{Les conseils de prud'hommes en France}

Les conseils de prud'hommes règlent «par voie de conciliation des différends qui peuvent s'élever à l'occasion de tout contrat de travail (...) entre les employeurs, ou leurs représentants, et les salariés qu'ils emploient. Ils jugent les litiges lorsque la conciliation n'a pas abouti $»{ }^{9}$ Les conseils de prud'hommes sont compétents pour les litiges individuels nés à l'occasion d'un contrat de travail de droit privé. Cela exclut donc les litiges mettant en jeu des intérêts collectifs (interruption d'une grève, lock-out, problèmes d'interprétation d'une convention collective ou d'un accord collectif entre une organisation syndicale et un employeur...). En tant que juridiction paritaire, les conseils

9. Code du Travail, art. L. 1411-1

10. Une réforme de 2008 a porté le nombre de conseils de prud'hommes à 210. Ils étaient auparavant 271. Pour une étude de l'impact de la réduction du nombre de conseils sur la demande de litiges et leur durée, voir Espinosa, Desrieux et Wan "Fewer Courts, less Justice? Evidence from the 2008 reform of French labor Courts », document de travail (2015). de prud'hommes regroupent deux collèges: un collège réunissant les représentants des employeurs, et un collège représentant celui des salariés.

Chaque conseil de prud'hommes se compose de cinq sections : agriculture, industrie, commerce, encadrement et activités diverses. C'est en général l'activité principale de l'entreprise qui permet de déterminer la section compétente. Quant au conseil compétent, il est généralement celui dans le ressort duquel se trouve l'établissement où est effectué le travail. Si le travail est réalisé en dehors de tout établissement (VRP, travailleurs à domicile...), la demande est portée devant le conseil de prud'hommes du domicile du salarié. On compte aujourd'hui 210 conseils de prud'hommes répartis dans tout le territoire français. ${ }^{10}$

\section{A. Les conseillers prud'homaux}

La justice prud'homale est rendue, non par des magistrats professionnels ayant la qualité de fonctionnaires d'État, mais par des juges élus tant par les salariés que par les employeurs et selon une logique de paritarisme. ${ }^{11}$ Généralisée en 1979, l'élection des conseillers prud'homaux s'est faite par un vote direct des électeurs, organisé tous les cinq ans. ${ }^{12}$ La dernière élection en date est de 2008: 14512 conseillers ont alors été élus. L'élection se fait au suffrage universel

11. Lorsqu'ils sont salariés dans une entreprise, les conseillers prud'homaux bénéficient d'un mandat propre leur permettant d'exercer leur fonction de magistrat élu.

12. Les règles de fonctionnement des élections prud'homales décrites dans ce paragraphe sont celles appliquées pendant notre période d'étude (1998-2012). Une réforme est actuellement en cours. 
direct, organisée pendant le temps de travail des salariés, le même jour sur tout le territoire. Le scrutin a lieu par liste, à la représentation proportionnelle intégrale, avec répartition des restes suivant la règle de la plus forte moyenne, sans panachage ni vote préférentiel. Le vote se tient par collège et par section. ${ }^{13}$ Sont électeurs tous les salariés, les employeurs et les demandeurs d'emploi, inscrits sur les listes électorales prud'homales, à condition de remplir certaines conditions. ${ }^{14}$ Les électeurs ne sont pas inscrits dans leur commune de résidence mais dans la commune de leur établissement de travail, selon le collège et la section auxquels ils sont rattachés. Les règles d'éligibilité prévoient d'être de nationalité française, être âgé de 21 ans au moins et n'être l'objet d'aucune interdiction, déchéance ou incapacité relative aux droits civiques. Une abstention croissante des électeurs a été observée au cours des années. Le taux de participation obtenu au scrutin de 2008 est de 25,63\% de votants sur l'ensemble du corps électoral. Plus précisément, le taux de participation a été de 25,48\% pour le collège salariés (18 683971 inscrits) et de $31,16 \%$ pour le collège employeurs (518 688 inscrits).

13. Rapport «Pour le renforcement de la légitimité de l'institution prud'homale: Quelle forme de désignation des conseillers prud'hommes? », M. Jacky RICHARD, Conseiller d'État M. Alexandre PASCAL, Inspecteur de I'Inspection générale des affaires sociales (IGAS), Rapport pour le Ministère du Travail, de la solidarité et de la fonction publique, Avril 2010.

14. Les électeurs doivent être âgés de 16 ans minimum ; être ou avoir été sous contrat de travail de droit privé français ou être
Les deux collèges (employeurs et salariés) ont montré des paysages sensiblement différents au cours des derniers scrutins. En effet, les principales fédérations représentant les employeurs ont majoritairement choisi de faire une liste commune. Au contraire, les syndicats salariés ont choisi de présenter des listes différentes lors des élections, et se sont donc fait concurrence au moment du vote. Parmi les cinq principaux syndicats salariés ayant présenté des listes, on distingue habituellement les syndicats «réformistes» (CFE-CGC, CFTC et CFDT) et les syndicats «non-réformistes » (CGT et FO). ${ }^{15}$ Dans les débats nationaux, ces derniers se caractérisent souvent par de plus fortes confrontations avec les fédérations patronales que les syndicats «réformistes».

Au cours des trois dernières élections, les résultats pour le collège salariés montrent que les syndicats non-réformistes obtiennent généralement entre 50 et $55 \%$ des voix, alors que les syndicats réformistes ont des scores établis entre 45 et $50 \%$ des voix. Le tableau 1 résume ces résultats (au niveau agrégé, c'est-à-dire national). employeur de personnes sous contrat de droit privé français. Les non ressortissants français ont la qualité d'électeur lorsqu'ils sont titulaires d'un contrat de travail régi par le droit français. Enfin, il ne faut pas être privé de ses droits civiques par l'effet d'une décision de justice pour pouvoir être électeur. 15. Le syndicalisme réformiste est parfois également dénommé «syndicalisme social-démocrate», et opposé au «syndicalisme révolutionnaire » ou « contestataire». 
Dossier Conseils de Prud'hommes, Syndicats et résolution des litiges : une analyse économique

Tableau 1. Les résultats des élections prud'homales

\begin{tabular}{|l|l|l|l|}
\hline Syndicat & \multicolumn{3}{|c|}{ Part des sièges obtenus en... } \\
\hline & \multicolumn{1}{|c|}{1997} & \multicolumn{1}{|c|}{2002} & \multicolumn{1}{c|}{2008} \\
\hline CGT & $34,6 \%$ & $35 \%$ & $39,7 \%$ \\
\hline FO & $22 \%$ & $18,3 \%$ & $16 \%$ \\
\hline CFDT & $32,2 \%$ & $31,9 \%$ & $26,9 \%$ \\
\hline CGC & $6,5 \%$ & $7,1 \%$ & $8,9 \%$ \\
\hline CFTC & $3,1 \%$ & $4,9 \%$ & $3,6 \%$ \\
\hline Autres & $1,6 \%$ & $2,6 \%$ & $4,9 \%$ \\
\hline
\end{tabular}

Source : Ministère du travail

\section{B. Le déroulement d'un litige}

Lorsque deux parties choisissent de porter leur conflit devant un conseil de prud'hommes, la première étape (sauf exceptions ${ }^{16}$ ) est celle de la conciliation. La conciliation est un préalable aux règlements des conflits du travail en audience non publique durant laquelle deux conseillers prud'homaux (un de chaque collège) tenteront d'aider les parties à résoudre leur conflit à l'amiable. Si la conciliation aboutit, cela évite le procès. Étant l'affaire des parties, elle ne peut jamais leur être imposée. En cas d'échec de la conciliation, l'affaire est renvoyée devant le bureau de jugement, si les parties ne souhaitent pas l'abandonner. Il

16. Les litiges dispensés de conciliation sont par exemple la requalification d'un CDD ou d'une mission d'intérim, la contestation de refus par l'employeur de l'octroi de certains congés (dont les congés de formation pour les représentants du personnel), le licenciement d'un administrateur élu par les salariés, ou encore les contentieux liés à une atteinte aux droits des personnes, à leur santé physique et mentale, ou aux libertés individuelles. existe un délai entre l'audience de conciliation et l'audience de jugement, afin de permettre aux parties de se mettre en état. ${ }^{17} \mathrm{Le}$ bureau de jugement est composé de deux conseillers issus du collège employeurs et deux conseillers issus du collège salariés. Lorsque les débats ont pris fin, les conseillers sont appelés à se prononcer sur la solution à donner au litige. Les délibérés sont secrets. Pour qu'un jugement soit rendu, il faut qu'une majorité de voix se dégage, c'est-àdire qu'au moins trois membres soient d'accord pour approuver la même solution au litige. Lorsqu'il y a deux voix contre deux, l'affaire est mise en départage. ${ }^{18}$ «En cas de départage, l'affaire est renvoyée devant (...)

17. Les parties échangent leurs arguments écrits et les pièces sur lesquelles elles se fondent. Les juges en prennent connaissance puis fixent une date d'audience. Ainsi, le dossier est «mis en état » d'être jugé.

18. Pour plus de détails sur les éléments de procédure, voir "Procès aux prud'hommes: de la saisine au jugement», V. Bardin-Fournairon et O. Barrault, coll. "pratiques d'experts», Groupe Revue Fiduciaire, 2013. 
le même bureau de jugement (...) présidé par un juge du tribunal d'instance dans le ressort duquel est situé le siège du conseil de prud'hommes ». ${ }^{19}$ Le renvoi en départage n'a pas à être motivé : ce n'est ni un jugement au fond ni une ordonnance mais un acte d'administration judiciaire non susceptible de recours. Comme pour le bureau de jugement, le prononcé du jugement rendu en départage doit être prononcé en audience publique.

\section{Raisonnement économique et stratégies des parties}

\section{A. Les déterminants de la négociation}

La littérature économique a cherché à établir des propositions sur les incitations des parties à négocier plutôt que d'aller devant les tribunaux, à partir de «modèles de litiges ». Ces modèles sont des constructions théoriques formalisées, permettant de rendre compte du comportement des parties. Ils proposent un cadre d'analyse simplifié, reposant sur l'hypothèse de rationalité des parties: elles prennent leurs décisions et agissent dès lors que le bénéfice de l'action est supérieur à son coût. Dans cette représentation simplifiée, on suppose la présence d'un demandeur et d'un défendeur. On suppose également deux étapes: les parties tentent tout d'abord de négocier, et si la négociation n'aboutit pas, alors elles portent leur conflit devant un tribunal. Dès lors, la négociation se fait «à l'ombre du droit»: les parties anticipent ce que pourraient être leurs gains ou leurs coûts si le conflit était porté au tribunal. Ces anticipations représentent «leur option de sortie », c'est-à-dire le gain ou le coût anticipé du procès. Ces options de sortie influencent le comportement des requérants pendant l'étape de négociation. En effet, l'idée générale est de considérer que les parties vont parvenir à une solution négociée si cela est dans l'intérêt de chacune d'elle. Autrement dit, le demandeur acceptera une compensation $S$ proposée pendant la négociation, si ce montant est supérieur ou égal à ce qu'il anticipe obtenir au procès. En ce qui concerne le défendant, la perspective du procès représente un coût, car il peut être potentiellement condamné à verser une compensation. Ainsi, il acceptera une solution négociée, si celle-ci prévoit qu'il doit verser un montant de compensation S inférieur ou égal au coût anticipé du procès. On peut donc déduire de ce raisonnement que les parties accepteront de négocier ensemble s'il existe un montant $S$ supérieur au gain net anticipé du procès pour le demandeur, et inférieur au coût total anticipé du procès pour le défendeur. Dès lors, tous les facteurs qui tendent à augmenter le coût du procès pour le défendeur, vont favoriser la négociation. Parmi ces coûts, nous retenons non seulement les frais financiers d'une action en justice, mais aussi tous les coûts dits «d'opportunité », c'est-à-dire le temps passé à constituer les dossiers de défense, à attendre, préparer et assister aux audiences. D'un point de vue économique, il s'agit bien de coûts dans la mesure où les par- 
ties auraient pu investir ce temps dans d'autres activités plus lucratives. Ainsi, lorsque les parties anticipent de longues durées de règlements des litiges, elles ont plus d'incitations à négocier en amont pour éviter de s'engager dans de longues procédures.

\section{B. L'application au conseil des prud'hommes}

Le raisonnement précédent peut s'appliquer au cas des conseils de prud'hommes. Si les parties anticipent que les conseils à majorité non-réformiste recourent plus souvent au départage car les conseillers ont plus de difficultés à trouver un accord (acceptation ou rejet de la demande) en bureau de jugement, ils anticipent que la durée du litige sera plus longue dans ces conseils. Pour éviter de s'engager dans des procédures trop longues et coûteuses, ils ont alors plus d'incitations à trouver une solution négociée, que ce soit dans le cadre du conseil de prud'hommes (i.e. la conciliation) ou bien en dehors du conseil (négociation informelle). Notre objectif est donc de voir si les parties négocient plus souvent lorsqu'elles sont confrontées à des conseils à majorité non-réformiste ou non.

Pour atteindre cet objectif, il nous faut procéder à une série de contrôles pour s'assurer que la plus grande incitation à négocier provient bien d'une anticipation plus longue de la durée de la procédure. Tout d'abord, il faut s'assurer que les conseils à majorité non-réformistes ont un recours plus fréquent au départage et donc des durées de litiges plus longues en moyenne du fait de ce recours plus fréquent au départage. De plus, il faut regarder si la composition syndicale n'influence pas d'autres variables comme le taux d'acceptation en bureau de jugement ou en départage. En effet, si cela était avéré, les parties pourraient anticiper une probabilité de victoire plus ou moins forte selon la composition du conseil, et leur attitude lors de la négociation pré-conseil s'en trouverait altérée. Enfin, il faut également tenir compte du nombre de litiges déposés devant chaque type de conseil de prud'hommes: on pourrait imaginer que certains conseils attirent plus de litiges que d'autres car des parties accepteraient de porter leur action en justice uniquement devant des conseils à majorité non-réformiste. Cela pourrait alors influencer la décision des parties de négocier ou non dans ces conseils.

En parallèle de ces contrôles, nous devons également tenir compte des autres facteurs susceptibles d'influencer les stratégies des parties ou les taux d'acceptation. Parmi ceux-ci, le fait d'être représenté, et le type de représentation (par un avocat ou un délégué syndical) peuvent avoir une influence. L'environnement macro-économique peut aussi être pris en compte, à l'instar des résultats proposés dans les contributions d'Ichino (2003) et Marinescu (2011). Il s'agit donc maintenant de voir si l'ensemble de ces facteurs ont une influence sur la probabilité qu'ont les parties à négocier, et sur la nature du jugement rendu (en bureau de jugement ou en formation de départage). 


\section{L'analyse empirique}

\section{A. Les données}

Afin de voir si le raisonnement établi précédemment a une validité empirique, c'est-àdire s'il se retrouve dans les faits, nous proposons une étude économétrique. Ce travail s'est appuyé sur une base de données fournie par le Ministère de la Justice délivrant des informations sur tous les litiges portés aux conseils de prud'hommes à partir de 1998, et terminés en 2012. Nous disposons donc de 1339496 cas (avec conciliation obligatoire) ouverts par des salariés. La plupart sont relatifs à des contestations de licenciements. ${ }^{20}$ Les informations dont nous disposons sur ces cas sont surtout la nature de leur issue (conciliation, acceptation ou rejet de la demande, avec ou sans départage). Nous savons dans quel conseil et dans quelle section le cas a été porté, mais nous ne connaissons pas l'identité des conseillers présents pendant les débats, ni le montant des compensations éventuellement versées. Le tableau 2 propose quelques statistiques descriptives sur l'issue de l'ensemble des litiges observés.

Tableau 2: Issues des litiges

\begin{tabular}{|l|c|c|}
\hline \multicolumn{1}{|c|}{ Issue du litige } & $\begin{array}{c}\text { Nombre } \\
\text { de litiges } \\
\text { concernés }\end{array}$ & $\begin{array}{c}\text { Pourcentage } \\
\text { sur l'ensemble } \\
\text { des litiges }\end{array}$ \\
\hline Conciliation & 180436 & $13,47 \%$ \\
\hline Abandon (après conciliation) & 331562 & $24,75 \%$ \\
\hline Acceptation de la demande en bureau de jugement & 514447 & $38,41 \%$ \\
\hline Rejet de la demande en bureau de jugement & 188762 & $14,09 \%$ \\
\hline Acceptation de la demande en départage & 86888 & $6,49 \%$ \\
\hline Rejet de la demande en départage & 37401 & $2,79 \%$ \\
\hline
\end{tabular}

Ce tableau montre qu'une forte proportion de litiges est tranchée en faveur du salarié par le bureau de jugement $(38,41 \%)$. Une faible part des litiges est conciliée, bien que cette part ne soit pas négligeable $(13,47 \%)$. Une proportion plus importante des litiges se

20. Voir les travaux de E. Serverin (dont Severin, E. and Valentin, J. (2009). Licenciement et Recours aux Prud'hommes, Questions de Mesure. In Gomel, B., Méda, D., and Severin, E., editors, trouve abandonnée après l'étape de conciliation $(24,75 \%)$. Enfin, le départage ne concerne que 8 à $10 \%$ de l'ensemble des litiges. Cependant, si on s'intéresse à la probabilité conditionnelle, c'est-à-dire à la probabilité d'aller au départage sachant que le litige

L'emploi en ruptures. Dalloz. ) et le numéro Infostat Justice no 105 (2009) pour une description des motifs des litiges portés aux conseils de prud'hommes. 
a déjà atteint le bureau de jugement, celle-ci avoisine un taux proche de $15 \%$ des litiges.

Grâce au Ministère du Travail, nous avons également pu accéder aux données sur les résultats des élections prud'homales dans chaque section de chaque conseil de prud'hommes, sur toute la période 1998 2012 (voir tableau 1). ${ }^{21}$ Sur cette période, trois élections ont déterminé la composition des conseils (1997, 2002, et 2008).

\section{B. La đémarche économétrique}

L'intérêt de l'analyse économétrique consiste à estimer la relation entre une variable $A$ et une variable $B$, tout en prenant en compte l'influence potentielle d'une série de variables C. Il s'agit de voir si les deux variables $\mathrm{A}$ et $\mathrm{B}$ sont corrélées entre elles, c'est-à-dire que la variation de l'une est associée à la variation de l'autre (dans le même sens ou en sens opposé). En ce qui concerne notre étude, l'objectif est d'expliquer ce qui détermine les différentes issues possibles des litiges (la conciliation, l'abandon, l'acceptation ou le rejet de la demande, le recours au départage). Pour «expliquer» ces variables, une attention particulière est portée sur la composition syndicale des conseils de prud'hommes (représentant ici notre variable «explicative»). Cet effet est isolé, tout en contrôlant pour un certain nombre d'autres facteurs, tels que le type de représentation du demandeur, du défendeur, le genre, le taux de chômage et le PIB. L'analyse économétrique

21. Nous remercions H. Fraisse, F. Kramarz et $C$. Prost de nous avoir transmis ces informations relatives aux élections prud'homales, collectées dans le cadre de leur article «Labor Disputes tient également compte de caractéristiques propres à chaque section de chaque conseil de prud'hommes: par exemple, il se peut que certaines caractéristiques de l'économie locale aient une incidence sur l'issue des litiges.

Pour tenir compte de ces effets, nous introduisons des «effets fixes par section de chaque conseil de prud'hommes », destinés à capturer les particularités locales qui ne varient pas au cours du temps. Ces effets capturent également les spécificités propres à chaque section (agriculture, industrie, encadrement, commerce et activités diverses). De la même façon, des effets fixes par année sont intégrés afin de tenir compte des caractéristiques propres à certaines années pouvant influencer l'issue du litige.

\section{Les résultats empiriques}

Pour mesurer la composition syndicale des sections dans les conseils de prud'hommes, différents indicateurs sont proposés dans notre étude. Nous nous concentrerons ici sur le plus simple d'entre eux, à savoir la proportion de sièges détenus par les syndicats nonréformistes (CFT et FO) sur l'ensemble des sièges de chaque section, dans chaque conseil. Les résultats de l'analyse économétrique montrent que cette composition syndicale a une influence significative sur l'issue des litiges. Plus la proportion de syndicats non-réformistes est élevée, plus on observe un succès de la conciliation, mais également un abandon des litiges et un recours

and Job flows», à paraître dans Industrial Labor Relations Review. 
au départage plus fréquents. Cependant, l'influence de la composition syndicale sur l'issue du jugement formulé soit par le bureau de jugement soit par la formation en départage n'apparaît pas significative : on ne peut donc pas établir une corrélation claire entre la composition syndicale des conseils et la nature du jugement rendu (acceptation ou rejet). Nos résultats montrent également que la composition syndicale des conseils de prud'hommes n'a pas d'effet sur le volume de litiges portés devant ces conseils.

Pour affiner nos résultats, nous proposons des techniques économétriques plus avancées en introduisant un modèle à double étape de sélection (triprobit). Celui-ci nous permet de tenir compte des caractéristiques inobservables des litiges, et principalement de leur «qualité ». Par qualité, nous entendons les preuves que peut détenir un plaignant et qui orientent favorablement la décision, ou bien la clarté du point de droit soulevé par le litige. Ainsi, un litige «de qualité élevée» pour le plaignant sera un litige qu'il a de fortes chances de remporter au vu des preuves à disposition et du problème rencontré. Puisque nous n'observons pas cette qualité des litiges, son effet est probablement inclus dans les marges d'erreurs de nos précédents résultats, appelés «termes d'erreur». Le traitement de ces derniers suggère que les litiges conciliés sont des litiges qui auraient eu de fortes chances d'être rejetés en bureau de jugement, alors que les litiges abandonnés auraient été

22. Nous entendons ici les litiges dont les preuves ne sont pas clairement en faveur ou défaveur du plaignant. Ce sont donc les litiges susceptibles de générer le plus de débats et d'interpréta- acceptés. Les parties semblent donc trouver une solution négociée en dehors de la cour pour ces cas de bonne qualité.

Pour résumer nos résultats, les conseils où les syndicats non-réformistes sont davantage présents et conduisent à plus de départage, ce qui illustre la plus grande difficulté de trouver un accord entre conseillers employeurs et salariés lors du bureau de jugement. Cela entraîne des durées de procédures plus longues, et

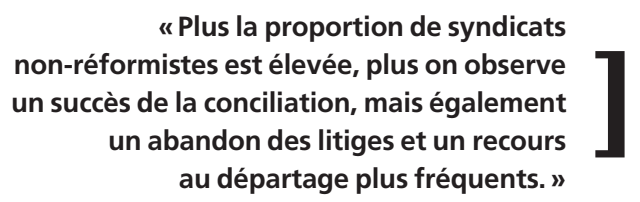

donnent donc davantage d'incitations aux parties à négocier en amont pour éviter le bureau de jugement. Les litiges de “bonne qualité" (favorables au salarié) sont davantage abandonnés, probablement au profit d'une négociation en dehors du conseil. Les litiges de "mauvaise qualité" (en défaveur du salarié) font eux davantage l'objet d'une conciliation. A la fois les litiges de très bonne et de très mauvaise qualité évitent donc le bureau de jugement. La qualité des litiges portés devant ce bureau des jugements reste donc inchangée en moyenne, d'où l'absence d'effet significatif de la composition du conseil sur la décision (acceptation ou rejet) prise par ce bureau. Cependant, un phénomène de polarisation a lieu entre conseillers employeurs et salariés: les cas de qualité “moyenne" 22 ne par-

tions des règles de droit, d'où un phénomène de polarisation: à la fois les conseillers salariés et employeurs sont moins enclins à faire des concessions. 
viennent pas à être décidés, d'où un recours plus fréquent au départage. ${ }^{23}$ Enfin, la composition syndicale des conseils n'influence ni le volume de litiges ni la décision finale sur le litige: nous ne repérons donc pas de différences importantes sur le nombre et la nature des litiges qui seraient causés par la couleur syndicale des conseils de prud'hommes.

\section{Conclusion et discussion}

Les résultats présentés ici sont une première étape dans la compréhension de l'influence de la composition syndicale sur l'issue des litiges. Plusieurs données demeurent manquantes et nous empêchent d'affiner nos résultats. Par exemple, nous ne disposons pas des montants de compensation que le demandeur peut avoir. Or la composition syndicale pourrait non seulement influencer les probabilités d'acceptation de la demande, mais aussi les montants reçus. Nous ne connaissons également pas les causes exactes du litige, alors qu'il pourrait être utile de savoir si la composition syndicale affecte tous les litiges de la même manière. Les votes individuels des conseillers ne sont également pas connus, ni les raisons ayant conduit au départage.

En dépit de ces limites, nos résultats peuvent être mis en perspective avec le débat actuel sur les juridictions de droit du

23. Rappelons ici que la composition syndicale $n$ 'influence pas la décision (acceptation ou rejet) du juge départiteur. Cela suggère que les conseils à majorité non-réformiste ne votent pas systématiquement en faveur du salarié: le juge départiteur se trouverait dans ce cas plus fréquemment confronté à des travail. Comme rappelé dans le rapport Lacabarats ${ }^{24}$, l'État a été régulièrement condamné pour dysfonctionnement de la justice prud'homale. A titre d'exemple, en 2013, il a été condamné 66 fois pour dysfonctionnement de la justice civile, pour un montant total de 1855311 euros. Cinquante et une de ces condamnations portaient sur un déni de justice en matière prud'homale. Il apparaît donc nécessaire de réformer cette justice, ce qui a récemment été mis en avant par certaines dispositions de la «loi Macron ». Parmi les sujets de discussion, la question du rôle des juges professionnels est de premier plan. Une certaine méfiance peut en effet se faire ressentir envers une justice exercée par des juges nonprofessionnels. A ce sujet, le rapport cité précédemment rappelle (p.17) que «dans le cadre des travaux menés sous l'égide du Conseil de l'Europe, qui constituent une référence essentielle pour la protection des droits fondamentaux et la qualité des systèmes judiciaires, le rapport d'évaluation adopté au mois de décembre 2013 à Strasbourg par le Groupe d'États contre la corruption (GRECO) recommande à la France de conduire une réforme non seulement pour les tribunaux de commerce, mais aussi pour les conseils de prud'hommes, "afin de renforcer l'indépendance, l'impartialité et l'intégrité des juges non professionnels". » Que peut apporter notre étude sur ce sujet?

litiges de mauvaise qualité, et devrait voter plus régulièrement en défaveur du salarié. Nous n'obtenons pas un tel résultat. 24. L'avenir des juridictions du travail: Vers un tribunal prud'homal du Xxpe siècle, A. Lacabarats, Rapport à Madame la Garde des Sceaux, Ministre de la Justice, Juillet 2014. 
Notre interprétation reste prudente. Nos résultats montrent qu'il n'y a pas de différences sur les taux d'acceptation des litiges entre les conseils à majorité non-réformiste et les autres: il n'y aurait donc pas de biais favorisant les salariés dans les conseils à majorité non-réformiste. Cependant, ces résultats peuvent cacher des situations variées: il se peut qu'aucun conseil (quelle que soit sa composition syndicale) n'exerce de biais en faveur des salariés; ou bien que tous les conseils exercent un même biais si bien qu'il n'existe pas de différences entre eux sur les taux d'acceptation. Nous ne sommes pas en mesure de différencier ces scénarios, ni même d'évaluer si un passage vers l'échevinage ou un système de juges professionnels changerait la situation. Nous soulignons simplement que la composition syndicale des conseils influence la probabilité du recours au départage ainsi que la stratégie des parties en matière de négociation: lorsqu'elles anticipent plus de confrontations entre les conseillers prudhommaux (dans les conseils à majorité non réformiste) et donc plus de départages, elles sont plus enclines à la négociation en amont.

De nombreuses études restent encore à mener pour mieux comprendre le fonctionnement de la justice prud'homale. La constitution d'une base de données avec davantage de renseignements nous permettrait de combler les lacunes évoquées précédemment (sur les motifs des litiges, le montant des compensations, les votes individuels etc...) et nous permettrait d'obtenir des proposi- tions plus précises. Le processus de décision des conseillers prud'homaux pourrait également être éclairé par d'autres travaux. Par exemple, déterminer si l'ordre du jour a une influence (c'est-à-dire le fait que l'audience d'un cas se tienne au début ou à la fin d'une série de litiges examinés) pourrait faire l'objet d'un autre travail empirique. D'autres sujets d'investigation pourraient concerner la réforme de 2008 ayant conduit à la suppression d'environ $20 \%$ des conseils de prud'hommes. Un travail a déjà été mené pour savoir dans quelle mesure cette suppression avait affecté le nombre d'affaires nouvelles et leur durée. Cependant, l'influence de cette réforme sur les flux entrants et sortants du marché du travail n'a pas encore été mesurée: en rendant potentiellement plus coûteuse la contestation d'un licenciement dans un conseil de prud'hommes (géographiquement plus éloigné), il se pourrait que la décision de licenciement ou d'embauche soit affectée. Tous ces thèmes ouvrent donc de nouvelles perspectives de travaux afin d'alimenter le dialogue entre juristes et économistes sur la justice prud'homale.

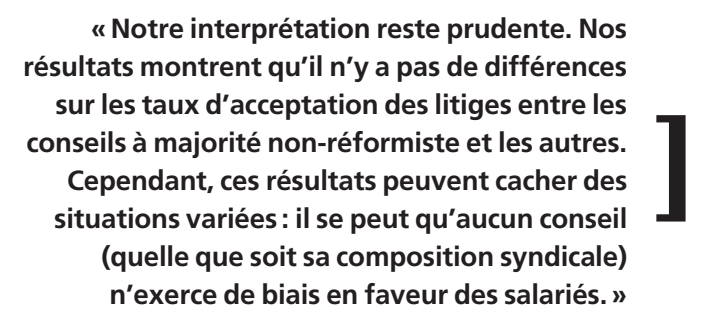

